\title{
Metabolic imprinting by leptin in early life to prevent obesity
}

\author{
C. Picó and A. Palou \\ Laboratory of Molecular Biology, Nutrition and Biotechnology (Nutrigenomics), University of the Balearic Islands (UIB) \\ and CIBER Fisiopatología de la Obesidad y Nutrición (CIBEROBN), Palma de Mallorca, Spain
}

Epidemiological data point that breastfeeding compared with infant formula feeding confers protection against several alterations later on in life and, particularly, against obesity and related medical complications. Leptin represents a candidate for the programming of lean phenotype, as suggested by (a) presence of leptin in breast-milk and its absence in infant formula; (b) when ingested during the suckling period it can be absorbed by the immature stomach and exert biological effects; (c) human studies showing a negative correlation between leptin concentration in breast-milk and body weight of infants; and (d) intervention studies in animals showing that leptin oral administration at physiological doses to neonate rats during whole lactation period has later positive effects, preventing animals from overweight/ obesity and other metabolic alterations, which are particularly associated with high-fat diet feeding. Although mechanisms involved in the protective effect of leptin treatment during lactation are not yet deciphered, results in animals show that leptin treatment during the suckling period promotes epigenetic modification in the POMC promoter with lasting effects on food intake and body weight, particularly when these animals were exposed to a HF diet. Thus, leptin may represent an essential nutrient during lactation in the protection against obesity and its metabolic-related disorders in later life.

This fact opens a new area of research on both the use of leptin in the design of more appropriate infant formula as well as the identification of potential factors influencing leptin levels in maternal milk, aspects of great relevance as strategies for the prevention of obesity from early stages of developments. 